\title{
Continuous Physiologic Monitoring: False Alarms and Overdiagnosis
}

\author{
Eric R. Coon, MD, MS*, H. Gilbert Welch, MD, MPH²
}

'Department of Pediatrics, University of Utah School of Medicine, Salt Lake City, Utah; ${ }^{2}$ Dartmouth Institute of Health Policy and Clinical Practice, Geisel School of Medicine, Hanover, New Hampshire.

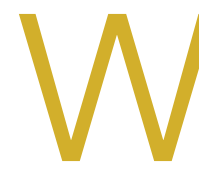

hat is the most common intervention to which hospitalized children are exposed? Acetaminophen? IV access? Phlebotomy? Or is it being connected to a monitor?

In a study conducted in five children's hospitals, Schondelmeyer et al found that exposure to continuous electronic physiologic monitoring was extremely common. During a selected 24-hour window of observation, nearly $100 \%$ of PICU and NICU patients and $26 \%-48 \%$ of medical-surgical patients were exposed to continuous monitoring. ${ }^{1}$ The latter is undoubtedly an underestimate given that monitoring periods less than 24 hours were not captured, patients may have been exposed before or after the 24-hour study window, and monitoring in the emergency department was not included.

The omnipresence of electronic physiologic monitoring in children's hospitals is striking, particularly because we know very little about its benefits. Outside of the perioperative period, there is a dearth of evidence demonstrating improved outcomes for hospitalized children as a result of continuous physiologic monitoring. Guidelines for the most common inpatient pediatric conditions do not advocate for continuous physiologic monitoring. Presumably, this practice has become so pervasive in the absence of a strong evidence base and guideline recommendations because it is a passive, seemingly innocuous intervention that continuously collects important components of the physical examination (after all, they are known as "vital" signs). It is tempting to assume that providing clinicians with this information will make patients safer.

The danger of routinely exposing children to an intervention for which the benefits are unproven is that the net effect of the intervention may be harm. What could be harmful? The simple act of monitoring is distressing to children; sticky electrode pads stuck to their skin and a tangle of wires that restrict their movement-all impeding physical activity and contact with loved ones.

Then, there are the alarms. Schondelmeyer et al report a staggering number of them: between 42 and 152 alarms per

\footnotetext{
*Address for correspondence: Eric R. Coon MD, MS, Department of Pediatrics, Division of Inpatient Medicine, University of Utah School of Medicine, Primary Children's Hospital, 100 North Mario Capecchi Dr, Salt Lake City, UT 84113; Telephone: (801) 662-3645; Fax: (801) 662-664; E-mail: eric.coon@hsc. utah.edu
}

Published online first April 25, 2018

Received: February 16, 2018; Accepted: February 23, 2018

๑ 2018 Society of Hospital Medicine DOI 10.12788/jhm.2974 monitored day on the floor; between 54 and 351 alarms in the intensive care units. The vast majority are false alarms, triggered by inappropriate preselected thresholds or displaced leads. This cacophony of noise only amplifies an already stressful environment for our patients-and their parents. Nurses and physicians are similarly stressed by alarms, not only by the noise but also by the frequent need to respond to them. The combination of frequent and largely unnecessary interruptions leads to alarm fatigue, whereby providers are desensitized to the alarms and may be slower to recognize a truly decompensating patient.

Continuous monitoring also risks overdiagnosis, the accurate detection of abnormalities that are not destined to cause problems, but nonetheless trigger interventions that can cause harm. ${ }^{2}$ Studies in adult populations have demonstrated that continuous monitoring can produce overdiagnosis. Repeated Cochrane reviews conclude that continuous electronic fetal monitoring during labor is associated with overdiagnosis of fetal distress-with attendant increase in cesarean sections without decreasing the risk for important neonatal outcomes such as cerebral palsy and mortality. ${ }^{3} \mathrm{~A}$ recent randomized trial of continuous pulmonary impedance monitoring intended to reduce readmission rates in patients with $\mathrm{CHF}$ instead found that continuous monitoring resulted in overdiagnosis of $\mathrm{CHF}$ exacerbations-paradoxically increasing hospital admission with no significant change in mortality (in fact, mortality was nominally higher in the monitoring group). ${ }^{4}$

Pediatric providers are probably no less susceptible to the impulse to act in the face of abnormalities detected by continuous monitoring. EKGs and electrolyte panels may be ordered in response to transient arrhythmias. Similarly, it is challenging for providers to watch a monitor flashing elevated respiratory rates in an otherwise healthy infant with bronchiolitis and not seek an escalation in care, including increased oxygen flow or transfer to a higher acuity unit. Although arrhythmia and respiratory rate alarms were common in Schondelmeyer et al's study, low oxygen level was far and away the most common alarm. Indeed, the poster child of pediatric overdiagnosis in the setting of electronic physiologic monitoring is hypoxemia. The present body of literature suggests that overreliance on pulse oximetry among patients with bronchiolitis increases admission rates to the hospital and prolongs length of stay, without a measurable improvement in morbidity or mortality. ${ }^{5}$

Few patients cared for at American children's hospitals will be discharged without exposure to prolonged periods of continuous physiologic monitoring. Undoubtedly, there are inpa- 
tients who benefit from this technology, such as children on mechanical ventilators. Unfortunately, there are also patients who are undoubtedly harmed by it. Greater understanding of which types of patients are more likely to benefit and which are more likely to be harmed is needed to determine whether continuous physiologic monitoring should remain our most common hospital intervention.

Disclosures: The authors have no financial relationships relevant to this article to disclose.

Funding: No external funding was secured for this study.

\section{References}

1. Schondelmeyer AC, Brady PW, Goel W, et al. Physiologic monitor alarm rates at 5 children's hospitals. J Hosp Med. 2018;13(5):396-398. Published online first April 25, 2018.

2. Welch HG, Schwartz L, Woloshin S. Overdiagnosed: Making people sick in the pursuit of health. Boston, Mass: Beacon Press; 2011.

3. Alfirevic Z, Devane D, Gyte GM, Cuthbert A. Continuous cardiotocography (CTG) as a form of electronic fetal monitoring (EFM) for fetal assessment during labour. Cochrane Database Syst Rev. 2017;2:Cd006066.

4. van Veldhuisen DJ, Braunschweig F, Conraads V, et al. Intrathoracic impedance monitoring, audible patient alerts, and outcome in patients with heart failure. Circulation. 2011;124:1719-1726.

5. Quinonez RA, Coon ER, Schroeder AR, Moyer VA. When technology creates uncertainty: pulse oximetry and overdiagnosis of hypoxaemia in bronchiolitis. BMJ. 2017;358:j3850. 